RUNNING HEAD: Measurement Invariance

\title{
Comparing Classic and Novel Approaches to Measurement Invariance
}

Zoe Magraw-Mickelson¹, Alejandro Hermida Carrillo², Chuma Owuamalam³ ${ }^{2}$ Maas Misha'ari Weerabangsa $^{3}$, and Mario Gollwitzer ${ }^{1}$

1 Social Psychology Chair, Department of Psychology, Ludwig-Maximilians-Universität München, Munich, Germany

2 Institute for Human Capital Management, Ludwig-Maximilians-Universität München, Munich, Germany

3 Faculty of Medicine \& Health Sciences, University of Nottingham Malaysia, Selangor, Malaysia

Correspondence: Zoe Magraw-Mickelson magraw-mickelson@psy.lmu.de 


\begin{abstract}
Measurement invariance (MI) is vital to any comparison of heterogeneous groups. With multiple-group confirmatory factor analysis (MG-CFA), which is the standard practice for testing MI, there are widely acknowledged limitations, especially with a large number of groups for which strict invariance is difficult or impossible to achieve. New methods, specifically Alignment optimization, gives increased flexibility and new opportunities to make comparisons across a large number of groups. This article compares MG-CFA with Alignment optimization for MI testing in three demonstrative studies. First, in a study of eight countries looking at eight different measures, the MG-CFA method found strict MI is problematic; only partial invariance was achievable and additionally required some of the countries to be excluded in some analysis. However, in using the Alignment optimization method there were not these drawbacks. Next, in a cross-validation reanalysis of existing data from five countries, we tested the reproducibility of conclusions from Alignment and MG-CFA analyses, finding only the results of the Alignment method reproducible. Finally, we show how to apply a recent extension of the Alignment optimization method, Alignment-within-CFA (AwC), to correlational analysis with samples from 30 countries. In a comparison of results with and without Alignment adjustments, we found some differences in conclusions drawn. This article illustrates the differences between methods as well as how to apply them to cross-cultural research.
\end{abstract}




\section{Comparing Classic and Novel Approaches to Measurement Invariance ${ }^{1}$}

The ability to compare groups is a bedrock principle of any cross-cultural research project or any research that wants to compare heterogeneous groups, be that across nations, genders, age groups, or time points. Researchers depend on statistical methods to answer questions of comparability, specifically measurement invariance, which is to ensure that participants with the same true-score on a latent variable respond in a similar manner to a manifestation of that variable, that is, without any systematic biases due to group memberships, age, or measurement occasion. Although there has been a growing recognition of the importance of establishing measurement invariance (MI), there are still challenges to be met for execution, namely what is acceptable as "partial invariance," and what to do if it is not established? Multiple-group confirmatory factor analysis (MG-CFA) is the most common method for testing MI, but this method has limits. With an increasing number of groups, MGCFA becomes increasingly impractical because it requires exact equivalence (all parameters, intercepts and factor loadings are exactly equal across all items) which is increasingly unlikely with more groups, and therefore, for practical reasons testing for equivalence is often neglected. The Alignment method is an alternative approach to MG-CFA. In the present article, we will explore the use of the Alignment method to make possible what has up to now been more difficult to achieve: to compare latent means across three or more groups. From this we will demonstrate how these methods can be applied in cross-cultural psychology.

This paper will first describe MG-CFA and contrast it to the Alignment method theoretically. Next, we will apply both methods to a set of data from eight countries across eight measures. We will use this example to reflect on similarities and differences between both methods and their results. Then, we will use a data set from five countries to look at the reproducibility of results from both methods. Finally, we will demonstrate the applicability of

\footnotetext{
${ }^{1}$ Link to Supplementary Online Materials, including tables, data and scripts: https://osf.io/ymq4p/?view only=936ee5181d304043b0a77641240653a2
} 
new extensions of the alignment method (i.e., alignment-within-CFA, AwC) which allow for confirmatory analyses and hypothesis testing beyond the establishment of invariance of a scale and mean comparisons across groups.

\section{Measurement Invariance: A Primer}

Establishing full measurement invariance of a psychological self-report measure (a "scale") validates the comparisons of groups by ensuring that the central parameters defining a measurement model - that is, the factor structure (i.e. number of factors), loadings (i.e. unit of a scale) or intercepts (i.e. original of the scale) - do not systematically depend on irrelevant aspects of the data collection, such as group, culture, or measurement occasions. Lack of MI can lead to erroneous conclusions, in that the observed mean differences are not due to latent mean differences but rather due to irrelevant differences between groups, such as response sets (Chen, 2008; van de Vijver \& Tanzer, 2004). Some reasons for the lack of MI include sampling, translation issues, different interpretation of the questions, and cultural difference in social desirability and acquiescence (Heath, Martin \& Sprekesen, 2009; van de Vijver \& Tanzer, 2004).

Given its importance for valid research practice, it is surprising how often MI is not tested in published research. In a review of cross-cultural research between 2008 and 2015 only $17 \%$ of articles published in the Journal of Cross Cultural Psychology and only 1\% of articles published in Personality and Social Psychology Bulletin assessed measurement equivalence (Boer, Hanke, \& He, 2018). Chen (2008) also found only 17\% of cross-cultural research studies between 1985 and 2005 in the Journal of Personality and Social Psychology tested measurement invariance. While some scholars have pointed to an inadequate education of the importance and execution of invariance testing (Steenkamp \& Baumgartner, 1998), one reason why measurement invariance is so often disregarded in cross-cultural research is that the methods used to scrutinize MI may often be difficult to apply or only feasible under certain circumstances. For instance, the traditional method of multiple-group confirmatory 
factor analysis (MG-CFA) is often unattainable for many groups (Byrne \& van de Vijver 2017; Kim, Cao, Wang \& Nguyen, 2017; Marsh et al., 2016; Rutkowski \& Svetina 2014). While the literature is not precise at what is meant by many groups, finding invariance becomes more difficult as the number of groups increases, as does the number of possible violations: in MG-CFA, the statistical null hypothesis of measurement invariance is defined as exact equivalence of all parameters (i.e., zero deviation) across groups. This null hypothesis might be too strict (for a comparable argument, see Cohen, 1994), so MG-CFA is likely to reject models that are "practically comparable" if the deviation of parameters across groups is non-zero (Lomazzi, 2018). When researchers have unique, many-group data sets, and want to publish their findings, exact measurement equivalence practices are frequently impossible and therefore prohibitive to doing a comparative analysis of groups. This dilemma was described by Byrne and van de Vijver (2017): “As a result, these limitations have remained a major impediment to advancing our substantive knowledge of cross-group differences within the context of a wide variety of disciplines and in the conduct of numerous important large-scale studies both nationally and cross-nationally" (p. 540). An example of this issue can be seen in that researchers failed to find invariance in Schwartz values' scale in the European Social Survey (e.g., Davidov, Schmidt, and Schwartz 2008), rendering its use extremely complicated (e.g., some items had to be averaged, not all dimensions were found etc.). However, the utility of this measure was redeemed when using alignment method (Cieciuch et al., 2018). Therefore, further establishing the functionality of new methods is of utmost importance. Multiple-group confirmatory analysis is first attributed to the seminal work of Jöreskog (1971) and later extended to test invariance of latent factor means (Sörbom, 1974). The MG-CFA method for MI is tested in steps: after assessing model fit for each group separately, model fit is established across groups, that is, configural invariance or factor form invariance, which, if found, shows that the same items are associated with the same latent factor. Next, metric or "weak" factorial invariance is tested, which assumes that the items 
have similar factor loadings across groups, which in turn allows for predictive relationships to be compared across groups. This is followed by scalar or "strong" invariance which, when established, means the item intercepts are equivalent across groups and is sufficient for factor mean comparisons. Following this is residual invariance or "strict" invariance which compares the equivalence of construct variance; however, this level is rarely tested because it is broadly agreed upon as not necessary for latent mean comparison (Cheung, \& Rensvold, 2000). At each step, the more restrictive model is compared to the previous, lesser restrictive model, and when a significantly worse fit is found based on previously established benchmarks, then invariance is rejected (see Meredith, 1993; Vandenberg \& Lance, 2000). Traditionally, the changes in the chi square statistic were used as an indicator. However, because chi square is found to be highly sensitive to sample size, it is recommended to use the change in CFI of -.01( $\triangle$ RMSEA $<0.015 ; \triangle$ SRMR $<0.03$, Cheung \& Rensvold, 2002; Chen, 2007; Meade et al., 2008). However, with the issues of multiple groups, some further relaxing for the restriction for scalar invariance has been recommended (Kim et al., 2017; Rutkowski \& Svetina, 2014).

While MG-CFA is the most common method for testing MI, it has been most widely used in the context of two groups (Kim et al., 2017; Rutkowski \& Svetina, 2014). There is an ongoing discussion in the literature and cross-cultural research field about the issues around how to handle a comparison of more than two to three groups. MG-CFA is now recognized as too stringent for numerous groups (in the context of large-scale cross-national studies, this usually refers to twenty or more groups, Byrne \& van de Vijver 2017; Kim et al., 2017; Marsh et al.; 2016, Rutkowski \& Svetina 2014). However, as it is the most normative standard for MI testing, it is a good benchmark to compare other methods to.

As mentioned above, the MG-CFA method is found to be impractical for a large number of groups because exact scalar invariance is often not achieved in practice. Even though this is widely acknowledged as an issue with large scale projects (20+ groups), it is 
also a problem with small and midsized (three or more) groups (Asparouhov \& Muthén, 2014; Marsh et al., 2016; van de Schoot, Kluytmans, Tummers, Lugtig, Hox, \& Muthen, 2013). Particularly, in a comparison between 3 to 10 countries (or heterogeneous groups), the literature is vague as to the utility of alternative methods for testing measurement invariance. When a research question depends on group mean comparisons, what options are there when one fails to find strict MI? Typical solutions involve excluding items or groups, limiting the analysis to subgroups of countries, and "partial" invariance (explained below). The former is not ideal, firstly, because by deleting items, one sacrifices content validity. Secondly, reducing the number of countries or the breadth of comparison dimensions excludes what might be vital information to the hypothesis at hand (Lomazzi, 2018). This is especially drastic when comparing a mid-range number of groups (see Davidov, Meuleman, Cieciuch, Schmidt, \& Billiet, 2014, for further stipulations on the possibilities with non-invariant data). Partial invariance occurs when only a subset of parameters is invariant, while another subset varies across groups. In Boer et al.'s (2017) review of literature in Journal of Cross Cultural Psychology mentioned above, out of the 64 studies in which the equivalence of the measurement model has been tested, 46 reported some form of partial invariances. The literature says that just one item with non-invariant intercept can lead to erroneous conclusions when observed mean differences (i.e. differences between groups on an aggregate measure) are interpreted (Meredith, 1993; Steinmetz, 2013). However, if item parameters are invariant for at least $50 \%$ of the items in the scale, one can compare latent means, that is, estimated means of the latent factor on which the items load (Byrne et al., 1989, Reise, Widaman, \& Pugh, 1993, Steenkamp \& Baumgartner, 1998, Steinmetz, 2013). Although this is the implication of some simulation studies that investigate the consequences of partial MI, there is also debate about the soundness of the stepwise strategy (explained below) and conflicting results in simulation studies (Marsh et al., 2018; Rensvold \& Cheung, 2001; Vandenberg, 2002; van de Schoot et al., 2013). The partial invariance stepwise procedure has 
an inherent risk of producing a model that deviates substantially from the true model because it depends on the post-hoc freeing of parameters. This added element of chance means the results might not be replicable (Lomazzi, 2018, Marsh et al., 2016; MacCallum, Roznowski \& Necowitz, 1992). As Marsh et al. (2018) explain, “...there is no guarantee that the stepwise selection process, based on freeing factor loadings and intercepts to achieve an acceptable goodness of fit, will facilitate this objective of providing unbiased means" (p. 12).

Due to the limitations of MG-CFA for a large number of groups, researchers have explored other methods, including multilevel confirmatory factor analysis (Davidov, Dülmer, Schlüter, Schmidt, \& Meuleman, 2012), multilevel factor mixture modeling, ESEM (Byrne \& van de Vijver, 2017), Bayesian approximation MI (Muthén \& Asparouhov, 2013a) and Alignment optimization (Asparouhov \& Muthén, 2014). Based on a review of these methods by Kim et al. (2017), the Alignment method was chosen as the best suited to be an alternative to look at MI across a mid-range number of groups.

\section{Alignment Optimization}

Alignment optimization was first introduced by Asparouhov and Muthén (2014) as an alternative to MG-CFA. The idea is that estimates of group factor means can be made without requiring exact MI, which means that Alignment optimization is a method of approximate rather than exact invariance. It is theorized that by taking some amount of non-invariance into account, Alignment optimization provides more reliable models (Byrne \& van de Vijver, 2017; Marsh et al., 2018). Furthermore, Alignment optimization starts with a common configural model that contains all groups for which the intercepts and loadings are unconstrained, rather than a baseline model for each group separately as with MG-CFA (Byrne \& van de Vijver, 2017). From the configural model, the process uses maximum likelihood (ML) to estimate an optimal set of measurement parameters and it computes approximations based on that. This optimization procedure involves applying a simplicity function that works similar to the rotation criteria in EFA; that is, it simplifies the 
interpretation while the fit is the same as the configural model, i.e. the unrotated model. This allows for differences in model parameter estimates by using the most relaxed, thus best fit model. Means and variances of the latent variable can be computed on the basis of the optimal model.

The execution is a two-step process: first, a configural model that represents the best fitting model among all groups is established while fixing the factor means to 0 and fixing variances to 1, without constraining the loadings and intercepts. Rather than assuming invariance, Alignment optimization operates with the assumption that there is a degree of non-invariance and the goal is to keep it to a minimum. In the next step, the factor means and variances are freely estimated and undergo an optimization process for every group factor mean and item parameter (see Asparouhov \& Muthén, 2014). Once the minimization point has been reached, the analysis compares factor means and factor variances across groups using a "post-estimation algorithm" and identifies each model parameter (i.e., loadings and intercepts) that is significantly different from the average of that parameter across all groups. This is done with multiple pairwise comparisons (adjusted for alpha inflation). If estimates are not significantly different they are connected, creating an invariant set to which subsequent groups' parameters are compared to, while those that are significantly different are flagged as noninvariant. The output provides the latent means based on this model as well as the parameters flagged as non-invariant (see Asparouhov \& Muthén, 2014, for a detailed description of the statistical equations for the process).

To our knowledge, there is only one simulation study that compares partial invariance with MG-CFA to Alignment. Marsh et al., (2018) found that the Alignment optimization method shows less bias for both scalar and partial scalar models. Our goal is to follow up this work with real data that compares the results of group-level latent mean comparisons under partial invariance found using MG-CFA or Alignment optimization. This is followed by a comparison of the replicability of the results of the different methods. A demonstration of 
how to apply the Alignment optimization method in cross-cultural research concludes this paper.

\section{Study 1}

In the first study we want to investigate the implications of using traditional MG-CFA, in comparison to Alignment optimization for testing mean differences between samples from different countries for eight measures. Up until now, research on the difference between MGCFA and Alignment optimization has been mainly based on simulation studies or situations, in which the number of groups was very large and MI testing using MG-CFA was feasible. That is, the analysis showed too many mis-specified parameters that needed to be adjusted so that partial invariance was also not feasible (Byrne \& van de Vijver, 2017). Therefore, this study looks at a medium number of countries for which partial invariance is a feasible alternative and uses the analysis derived from the Alignment optimization method to compare the two methods in this context.

\section{Methods}

\section{Samples}

The data for this study is a secondary analysis on materials collected for an independent project. This multinational project involved researchers in eight countries that were all tasked with finding participants in their respective countries. The aim was to collect 200 participants per country but final participant numbers were varied due to the individual situation of the data collection location. In the case of the German sample, it was not possible to get more participants in the time frame allotted because recruitment depended on the size of the student sample pool at the time of data collection. The sample sizes, age range, and gender distribution can be seen in Table 1. All research groups were instructed to follow the translation-back translation process from the original English materials (preregistration document available at: https://doi.org/10.17605/OSF.IO/8MZCN). All participants completed the material on an online platform. The sample from Germany was collected by the first 
author. Participants were psychology Bachelor students who received course credits for their participation.

Table 1

Sample Information

\begin{tabular}{lcccc}
\hline \multicolumn{1}{c}{ Country } & Sample size & Age mean & age SD & \% female \\
\hline 1. USA & $\mathrm{N}=130$ & 26,01 & 5,40 & 53,4 \\
2. UK & $\mathrm{N}=294$ & 19,13 & 2,61 & 84,7 \\
3. Canada & $\mathrm{N}=72$ & 25,96 & 4,83 & 40,8 \\
4. Germany & $\mathrm{N}=116$ & 21,75 & 3,80 & 75,9 \\
5. India & $\mathrm{N}=177$ & 20,90 & 4,59 & 52,0 \\
6. Malaysia & $\mathrm{N}=169$ & 19,35 & 2,20 & 47,6 \\
7. Nigeria & $\mathrm{N}=205$ & 21,92 & 3,35 & 49,0 \\
8. Peru & $\mathrm{N}=92$ & 23,61 & 4,27 & 51,1 \\
\hline
\end{tabular}

\section{Measures}

The eight measures we analyzed for the present study are below:

Horizontal/Vertical Individualism/Collectivism Scale. 6-point Likert Scale $(1=$ Strongly Disagree; 6 = Strongly Agree) 4 items each.

Horizontal Collectivism (HC). Measures the degree to which people see themselves as similar to other people and emphasize common goals as well as interdependence (e.g. "To me, pleasure is spending time with others.").

Vertical Collectivism (VC). Measures the degree to which people emphasize the importance of the group and are willing to go along with the authority of the group even when it does not fit one's own interests (e.g., "Family members should stick together no matter what sacrifices are required.”).

Horizontal Individualism (HI). Measures the degree to which people value both equality and freedom and want to be unique (e.g., "I'd rather depend on myself than others.")

Vertical Individualism (VI). Measures the degree to which people want to be distinguished in order to gain status and value freedom over equality (e.g., "Competition is the law of nature," Triandis \& Gelfand, 1998). 
Social Dominance Ordination SDO-6 Scale. (Hierarchy Items) - 7-point Likert Scale $(1=$ Strongly Disagree; $7=$ Strongly Agree $), 8$ items. SDO measures one's motivation for social hierarchy and group-based dominance (e.g., "It is probably a good thing that certain groups are at the top and other groups are at the bottom," Pratto et al., 2000).

Right Wing Authoritarianism (RWA) short scale. 7-point Likert Scale (1 = Strongly Disagree; 7 = Strongly Agree), 7 items. RWA measures the belief in the uncritical subjection to authority and defense of authoritative norms (e.g., "Our country desperately needs a mighty leader who will do what has to be done to destroy the radical new ways and sinfulness that are ruining us;” Manganelli, Rattazzi, Bobbio, \& Canova, 2007).

Victim Sensitivity \& Observer Sensitivity (Justice Sensitivity subscales). 6-point Likert Scale ( 1 = Strongly Disagree; 6 = Strongly Agree $), 10$ items each.

Victim Sensitivity (VS). Measures sensitivity to being the victim of injustice, (e.g., "It bothers me when others receive something that ought to be mine").

Observer Sensitivity (OS). Measures sensitivity to injustice toward others (e.g., "I am upset when someone does not get a reward he/she has earned," Schmitt et al., 2005). The correlation between the measures for each country can be found in SOM (S1a-h).

\section{Analysis Strategy}

$M G-C F A$. For the multiple group confirmatory factor analysis, we followed a stepwise approach of establishing measurement invariance for each of the scales (see Meredith, 1993; Vandenberg \& Lance, 2000). We completed this analysis with lavaan (Rosseel, 2012) and semTools for R (Jorgensen, Pornprasertmanit, Schoemann, \& Rosseel, 2018). We looked at the fit indices that are usually reported in the literature and used values for acceptable model fit that have been suggested by Marsh, Hau \& Wen (2004), however, important for our analysis is the comparative relative fit at each level of invariance (Marsh et al., 2018). Here, we focused on a change of CFI of $<.01$ to represent acceptable difference in fit. 
If scalar invariance was not achieved, we proceeded to test for partial invariance. For partial invariance, we followed the stepwise approach by using a Lagrange multiplier (score) test to obtain the expected change in model fit if each constrained parameter is estimated freely, and then freeing one parameter at a time, starting with the largest value (Byrne, Shavelson, \& Muthén, 1989; Steenkamp \& Baumgartner, 1998). For each parameter freed, we again checked the comparative fit. Even though some of the MI literature states that two invariant indicators are enough to make meaningful comparisons (Cieciuch et al., 2018), practitioners often follow other rules when deciding how many items they can compromise when forming a scale. For example, on his highly cited paper on missing data, Graham (2009) describes how scale means should never contain less than $50 \%$ of the original number of items. Therefore, we repeated this process until a maximum of $50 \%$ of the parameters of the items were freed. Given that deleting items with noninvariant parameters is widespread practice in cross-cultural research when partial invariance is found, we deemed this as a threshold closer to actual research practice. If partial invariance was still not established, we began the process again by excluding countries with high modification indices or high chisquare values in the strict invariance model. Once we established partial invariance at the scalar level for all measures, we used these models to calculate the latent means and compared them with Germany as the reference group. We used Germany as the reference group because this was the sample collected by the primary author and where some of the scales were developed (i.e. VS and OS).

Alignment. Using Mplus version 8.1 (Muthén \& Muthén, 2018) and the syntax in the supplementary online materials, we followed guidelines from Asparouhov and Muthén (2014) for Alignment optimization, imputing each measure in a separate analysis. We used FIXED alignment rather than FREE as recommended by Marsh et al., (2018), Kim et al., (2017), and Jang et al., (2017). The FIXED option fixes the latent means of one group, in this case 
Germany to 0 and variance to 1, thus serving as the reference group (see Asparouhov and Muthén, 2014, for a detailed discussion on the difference between FIXED and FREE options). Research has been mixed on the degree of acceptable non-invariant parameters with the Alignment method that still allows a valid comparison. In the original simulation study for the Alignment method, Asparouhov and Muthén (2014) mentioned 20\% non-invariant parameters as acceptable; later, Muthén and Asparouhov (2014) adjusted that to 25\%. Flake \& McCoach (2018) stipulated there should be less than 29\% non-invariant parameters. However, as this is a relatively new field, Muthén and Asparouhov (2014) recommended when above $25 \%$, a Monte Carlo simulation should supplement the judgement of acceptability. Following this recommendation, Lomazzi (2018) found $21 \%$ acceptable. However, this is an ongoing, open question for Alignment optimization. Using the models resulting from the invariance testing by both methods, we compared the composite and latent means.

\section{Results}

The results of the MG-CFA invariance testing can be seen in Tables 3-7. For each measure, first step is the single group solution and initial test of full invariance followed by the results for partial invariance. The results show that we found metric invariance for all measures $^{2}$ but were unable to find scalar invariance. However, we did find partial scalar invariance with $\mathrm{HC} / \mathrm{VC} / \mathrm{HI} / \mathrm{VI}$ and SDO (the necessary intercepts freed for the partial model are listed in the Table). For VS, OS, and RWA, partial scalar invariance was only found for a subset of countries (also listed in Tables 2-6- of SOM).

For Alignment optimization, we first checked the overall degree of non-invariance for each scale. As mentioned above, the existing guidelines are that $25 \%$ of non-invariance is problematic or more strictly that those above $20 \%$ should require a closer look. None of our

\footnotetext{
${ }^{2}$ In the SDO scale the CFI difference was -.0104 on the metric vs. configural invariance model. We decided to not pursue weak invariance further by freeing item loadings in this scale because the goal of this study is to compare means across countries, which are mainly affected by noninvariance in the intercepts (Steinmetz, 2013).
} 
scales came close to this number. The highest degree of non-invariance across parameters was $2 \%$ of intercepts for the SDO scale. Therefore, we concluded that using Alignment optimization method invariance is established for all measures (see Tables 7-11 of SOM).

\section{Comparison of mean differences}

Table 2 shows a comparison of the means and the significance of mean differences. First, listed in the table are the simple composite means for each measure for each country followed by the latent means found with MG-CFA scalar partial invariance and finally the latent means from the Alignment optimization. As scalar invariance was not established for any of the scales, the composite scores provide an invalid comparison (Meredith, 1993; Steinmetz, 2013). However, these means provide reference for the degree to which the composite means may be inaccurate. Although there were some shifts in the rank order of the countries between the composite mean, MG-CFA latent means and Alignment latent means, those position shifts were not significant. However, there were numerous discrepancies in whether the mean differences between countries reached significance. This is true for both the comparison of the composite means and latent means, and the comparison between the latent means achieved by different methods. See Table 13 for all comparisons and differences in significance. Overall, there are more significant differences found when comparing the raw composite score to the MG-CFA latent means than when comparing them to the Alignment latent means. This points to a systematic directionality of the bias in the raw composite scores, in that mean differences are inflated between countries. 
Table 2

Mean comparisons 8 countries 8 scales

\begin{tabular}{|c|c|c|c|c|c|c|c|c|c|}
\hline 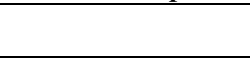 & \multicolumn{3}{|c|}{ RAW-Composite } & \multicolumn{3}{|l|}{ MG-CFA } & \multicolumn{3}{|c|}{ ALIGNMENT } \\
\hline & & $\begin{array}{l}\text { Factor } \\
\text { Mean }\end{array}$ & $\begin{array}{l}\text { Groups With } \\
\text { Significantly } \\
\text { Smaller Factor } \\
\text { Mean }\end{array}$ & $\begin{array}{l}\mathrm{HI} 1 \sim 1, \mathrm{HI} 2 \sim 1, \\
\mathrm{VI} 4 \sim 1, \mathrm{VC} 1 \sim 1, \\
\mathrm{HC} 1 \sim 1, \mathrm{VC} 2 \sim 1, \\
\mathrm{VI} 1 \sim 1\end{array}$ & $\begin{array}{l}\text { Factor } \\
\text { Mean }\end{array}$ & $\begin{array}{l}\text { Groups With } \\
\text { Significantly } \\
\text { Smaller Factor } \\
\text { Mean }\end{array}$ & & $\begin{array}{l}\text { Factor } \\
\text { Mean }\end{array}$ & $\begin{array}{l}\text { Groups With } \\
\text { Significantly } \\
\text { Smaller Factor } \\
\text { Mean }\end{array}$ \\
\hline \multirow{8}{*}{$\begin{array}{l}\text { Horizontal } \\
\text { Collectivism }\end{array}$} & 8. Peru & 4,85 & $6,5,3,1$ & 7. Nigeria & 0,119 & $3,5,2,6,1$ & 8. Peru & 0,172 & $6,5,3,1$ \\
\hline & 7. Nigeria & 4,77 & $6,5,3,1$ & 8. Peru & 0,056 & $3,5,1$ & 7. Nigeria & 0,149 & $6,5,3,1$ \\
\hline & 2. UK & 4,68 & 1 & 4. Germany & 0,000 & 3,1 & 4. Germany & 0,000 & \\
\hline & 4. Germany & 4,68 & & 2. UK & $-0,149$ & 1 & 2. UK & $-0,005$ & 1 \\
\hline & 6. Malaysia & 4,59 & & 6. Malaysia & $-0,175$ & 1 & 6. Malaysia & $-0,147$ & \\
\hline & 5. India & 4,55 & & 5. India & $-0,256$ & & 5. India & $-0,188$ & \\
\hline & 3. Canada & 4,48 & & 3. Canada & $-0,336$ & & 3. Canada & $-0,221$ & \\
\hline & 1. USA & 4,47 & & 1. USA & $-0,480$ & & 1. USA & $-0,286$ & \\
\hline \multirow{8}{*}{$\begin{array}{l}\text { Vertical } \\
\text { Collectivism }\end{array}$} & 7. Nigeria & 4,96 & $6,5,8,4,3,2,1$ & 7. Nigeria & 0,940 & $4,3,5,2,6,8,1$ & 7. Nigeria & 0,747 & $5,2,4,1,3,8$ \\
\hline & 6. Malaysia & 4,66 & $5,8,4,3,2,1$ & 6. Malaysia & 0,466 & $4,3,2,8,1$ & 6. Malaysia & 0,464 & $2,4,1,3,8$ \\
\hline & 5. India & 4,43 & $1,8,3$ & 5. India & 0,176 & 3,1 & 5. India & 0,244 & $1,3,8$ \\
\hline & 2. UK & 4,30 & $1,8,3$ & 4. Germany & 0,000 & 3,1 & 2. UK & 0,032 & $1,3,8$ \\
\hline & 4. Germany & 4,28 & $1,8,3$ & 2. UK & $-0,048$ & 3,1 & 4. Germany & 0,000 & $1,3,8$ \\
\hline & 1. USA & 3,97 & & 8. Peru & $-0,115$ & 1 & 1. USA & $-0,382$ & \\
\hline & 8. Peru & 3,93 & & 3. Canada & $-0,504$ & & 8. Peru & $-0,462$ & \\
\hline & 3. Canada & 3,91 & & 1. USA & $-0,545$ & & 3. Canada & $-0,533$ & \\
\hline \multirow{8}{*}{$\begin{array}{l}\text { Horizontal } \\
\text { Individualism }\end{array}$} & 3. Canada & 5,03 & $5,8,2,6,4$ & 7. Nigeria & 1,201 & $5,8,2,6,4$ & 7. Nigeria & 0,762 & $2,6,4$ \\
\hline & 7. Nigeria & 4,98 & $5,8,2,6,4$ & 3. Canada & 1,398 & $5,8,2,6,4$ & 3. Canada & 0,711 & $2,6,4$ \\
\hline & 1. USA & 4,88 & $8,2,6,4$ & 1. USA & 1,227 & $5,8,2,6,4$ & 1. USA & 0,651 & $2,6,4$ \\
\hline & 5. India & 4,69 & 6,4 & 5. India & 0,908 & $2,6,4$ & 8. Peru & 0,611 & 6,4 \\
\hline & 8. Peru & 4,63 & 6,4 & 8. Peru & 0,779 & 4 & 5. India & 0,441 & 6,4 \\
\hline & 2. UK & 4,57 & 6,4 & 2. UK & 0,632 & 4 & 2. UK & 0,313 & 4 \\
\hline & 6. Malaysia & 4,36 & & 6. Malaysia & 0,478 & 4 & 6. Malaysia & 0,084 & \\
\hline & 4. Germany & 4,23 & & 4. Germany & 0,000 & & 4. Germany & 0,000 & \\
\hline
\end{tabular}




\begin{tabular}{|c|c|c|c|c|c|c|c|c|c|}
\hline \multirow{10}{*}{$\begin{array}{l}\text { Vertical } \\
\text { Individualism }\end{array}$} & 7. Nigeria & 4,48 & $5,6,8,3,1,2,4$ & 7. Nigeria & 1,688 & $5,6,8,3,1,2,4$ & 7. Nigeria & 1,834 & $5,6,3,8,1,2,4$ \\
\hline & 5. India & 3,74 & $3,1,2,4$ & 5. India & 0,960 & $1,2,4$ & 5. India & 1,015 & $8,1,2,4$ \\
\hline & 6. Malaysia & 3,65 & $1,2,4$ & 6. Malaysia & 0,879 & $1,2,4$ & 6. Malaysia & 1,012 & $1,2,4$ \\
\hline & 8. Peru & 3,51 & 4,2 & 8. Peru & 0,695 & 2,4 & 3. Canada & 0,729 & 4 \\
\hline & 3. Canada & 3,37 & 4 & 3. Canada & 0,694 & 4 & 8. Peru & 0,572 & 4 \\
\hline & 1. USA & 3,30 & 4 & 1. USA & 0,562 & 4 & 1. USA & 0,545 & 4 \\
\hline & 2. UK & 3,23 & 4 & 2. UK & 0,395 & 4 & 2. UK & 0,430 & 4 \\
\hline & 4. Germany & 2,89 & & 4. Germany & 0,000 & & 4. Germany & 0,000 & \\
\hline & RAW-Composite & & & MG-CFA & & & ALIGNMENT & & \\
\hline & & $\begin{array}{l}\text { Factor } \\
\text { Mean }\end{array}$ & $\begin{array}{l}\text { Groups With } \\
\text { Significantly } \\
\text { Smaller Factor } \\
\text { Mean }\end{array}$ & $\begin{array}{l}\text {-USA, Nigeria, and } \\
\text { Peru; SDO08 } \sim 1, \\
\text { SDO05 } \sim 1, \text { SDO04 } \\
\sim 1, \text { SDO07 } \sim 1\end{array}$ & $\begin{array}{l}\text { Factor } \\
\text { Mean }\end{array}$ & $\begin{array}{l}\text { Groups With } \\
\text { Significantly } \\
\text { Smaller Factor } \\
\text { Mean }\end{array}$ & & $\begin{array}{l}\text { Factor } \\
\text { Mean }\end{array}$ & $\begin{array}{l}\text { Groups With } \\
\text { Significantly } \\
\text { Smaller Factor } \\
\text { Mean }\end{array}$ \\
\hline \multirow{10}{*}{$\begin{array}{l}\text { Social } \\
\text { Dominance } \\
\text { Ordination }\end{array}$} & 7. Nigeria & 3,75 & $6,5,2,3,8,1,4$ & 6. Malaysia & 0,934 & $2,3,4$ & 7. Nigeria & 1,297 & $5,2,8,3,1,4$ \\
\hline & 6. Malaysia & 3,31 & $2,3,8,1,4$ & 5. India & 0,768 & $2,3,4$ & 6. Malaysia & 1,117 & $2,8,3,1,4$ \\
\hline & 5. India & 3,21 & $2,3,8,1,4$ & 2. UK & 0,373 & 4 & 5. India & 0,925 & $2,8,3,1,4$ \\
\hline & 2. UK & 2,70 & 4 & 3. Canada & 0,288 & & 2. UK & 0,452 & 4 \\
\hline & 3. Canada & 2,64 & 4 & 4. Germany & 0,000 & & 8. Peru & 0,403 & 4 \\
\hline & 8. Peru & 2,57 & 4 & & & & 3. Canada & 0,380 & \\
\hline & 1. USA & 2,51 & 4 & & & & 1. USA & 0,314 & \\
\hline & 4. Germany & 2,10 & & & & & 4. Germany & 0,000 & \\
\hline & RAW-Composite & & & MG-CFA & & & ALIGNMENT & & \\
\hline & & $\begin{array}{l}\text { Factor } \\
\text { Mean }\end{array}$ & $\begin{array}{l}\text { Groups With } \\
\text { Significantly } \\
\text { Smaller Factor } \\
\text { Mean }\end{array}$ & $\begin{array}{l}\text {-India and Malaysia; } \\
\text { RWA06 } ~ 1, \\
\text { RWA01 } \sim 1, \\
\text { RWA04 } \sim 1 \\
\text { RWA02 } ~ 1\end{array}$ & $\begin{array}{l}\text { Factor } \\
\text { Mean }\end{array}$ & $\begin{array}{l}\text { Groups With } \\
\text { Significantly } \\
\text { Smaller Factor } \\
\text { Mean }\end{array}$ & & $\begin{array}{l}\text { Factor } \\
\text { Mean }\end{array}$ & $\begin{array}{l}\text { Groups With } \\
\text { Significantly } \\
\text { Smaller Factor } \\
\text { Mean }\end{array}$ \\
\hline \multirow{4}{*}{$\begin{array}{l}\text { Right Wing } \\
\text { Authoritarianism }\end{array}$} & 7. Nigeria & 5,43 & $6,5,2,8,1,3,4$ & 3. Canada & 0,454 & $4,3,2,8,1$ & 7. Nigeria & 2,666 & $6,5,8,2,1,3,4$ \\
\hline & 6. Malaysia & 4,71 & $2,8,1,3,4$ & 4. Germany & 0,000 & $1,3,4$ & 6. Malaysia & 1,876 & $8,2,1,3,4$ \\
\hline & 5. India & 4,47 & $2,8,1,3,4$ & 7. Nigeria & 2,858 & $1,3,4$ & 5. India & 1,687 & $2,1,3,4$ \\
\hline & 2. UK & 3,44 & $1,3,4$ & 8. Peru & 1,138 & 4 & 8. Peru & 0,919 & $1,3,4$ \\
\hline
\end{tabular}




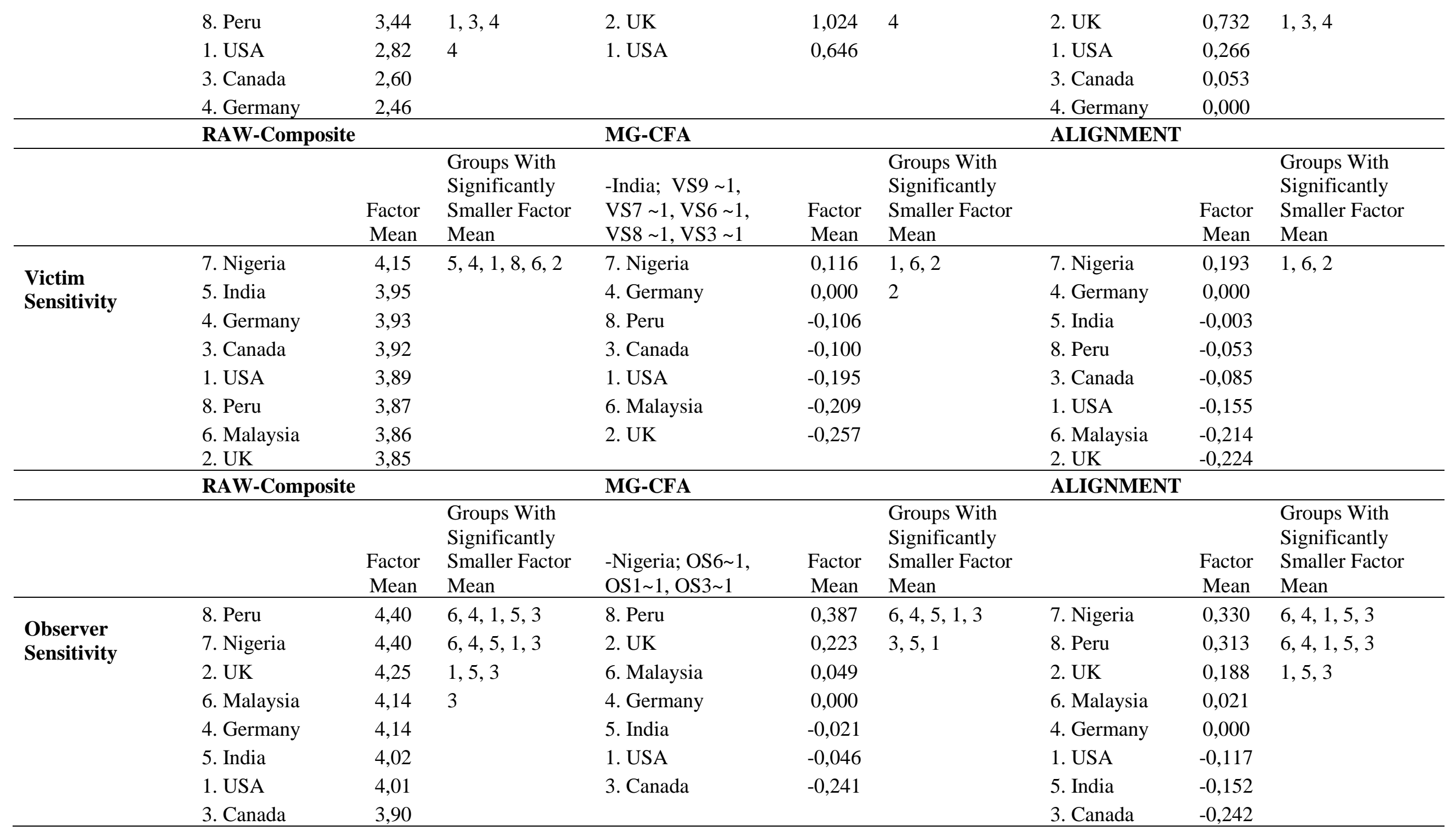




\section{Discussion}

This study employed data collected in eight different cultures to compare the utility of two different methods used to test measurement invariance. The literature on measurement equivalence points clearly to the problem that the lack of scalar MI can lead to erroneous conclusions when comparing composite means. Lacking the necessary scalar invariance, we looked at the latent means derived from two methods. In the case of this data set, the mean differences from the raw scores was inflated, both methods showed fewer mean differences when equivalency was ensured. Past research has extensively discussed the value of "partial" invariance for comparing latent means, although it provides an option when there is no scalar invariance, we added to this debate by finding differing results between latent mean comparisons using MG-CFA partial invariance and Alignment optimization. In Marsh et al. (2018), the degree of difference in significance between groups comparing latent means estimated on the basis of MG-CFA (with partial invariance) compared to on the basis of Alignment also shifted, but the direction depended on the measure. Simulation studies point to evidence that the relative bias of partial MG-CFA compared to approximate methods is sensitive to the number and size of differences in intercepts as well as the sample size (Marsh et al., 2018; van de Schoot et al., 2013). We follow this up by demonstrating, with real data, that the methods do not yield the same results, which opens the question of which method produces the true results. The most important note is of the utility of partial invariance from a practical standpoint, MG-CFA limits the analysis by requires the exclusion of some samples, while Alignment optimization does not.

For Studies 2 and 3 we continued with real data sets, using published data to further add to the picture of the differences between the methods. First, we add to the argument that one method is favored over another by testing the reproducibility of results. Second, we apply the knowledge of these differing methods to research questions of interest. 


\section{Study 2}

Study 1 demonstrated there are limitations to using MG-CFA compared to Alignment optimization when analyzing real data from a moderate number of countries, namely, partial invariance was required and the need to exclude some samples from the analysis. Notably, results in Study 1 are concurrent with the idea that partial invariance is problematic, in part because the stepwise procedure is post-hoc and there is no guarantee that the process (based on freeing parameters to achieve acceptable fit) will identify the true model and unbiased means (Marsh et al., 2018). These limitations could lead to the lack of replication for real data: to the best of our knowledge, no studies have looked at reproducibility of results with real data using these methods. If the scales are in fact comparable, we should find invariance with new data collected in similar samples. In study 2, we aim to give further consideration to this question by testing the reproducibility of result of MI testing using the two methods. We investigate this point by doing a cross-validation analysis of invariance, which involves randomly dividing the data in two and comparing the results for both MG-CFA and the Alignment optimization method. Theoretically, the conclusion based on each half of the data should be the same for both the methods of invariance testing.

\section{Sample}

The data for this exercise is the reanalysis of publicly available data collected as part of the Many Labs 2 project (Klein et al., 2018). We decided to use this project because, first, the data set is an open and widely available largescale cross-cultural research project. Second, such a project, a well-designed replication, is a suitable context for a robust test of the reproducibility of findings using different methods. The Many Labs 2 project aimed to replicate 28 classic studies across 125 samples from 36 countries and territories. However, for our analysis we grouped samples by country and only included those with at least 300 participants, in order to have a sufficiently large sample size for the random split. Five 
country groups fitted this criterion. The countries and total sample size can be found on Table 3.

Table 3

Sample information

\begin{tabular}{lc}
\hline Country & Sample size \\
\hline 1. Canada & 602 \\
2. China & 396 \\
3. India & 360 \\
4. Netherlands & 497 \\
5. USA & 2055 \\
\hline
\end{tabular}

\section{Measures}

To test for measurement invariance, we identified study number 12. Sociometric status and well-being (Anderson et al., 2012, Study 3), as suitable for our analysis, that is, it included a measure using a Likert scale with more than three items. That is, a seven-item scale of subjective well being measured on a 7-point scale from Likert Scale ( 1 = Strongly Disagree; 7 $=$ Strongly Agree) .

\section{Analysis Strategy}

First, in order test the reproducibility of results we randomly split the data for each sample. For our test of measurement invariance, we followed the sample analysis plan as outlined in Study 1 for each half of the data independently. For MG-CFA we followed the same cut-off recommendation and partial invariance procedure as outlined in Study 1. For the Alignment method analysis, we again used the FIXED option, in this case the USA serving as the reference group (all scripts can be found in SOM).

\section{Results}

The results of the MG-CFA invariance testing can be seen in Table 4. For each measure, the initial test of full invariance is followed by the results for partial invariance. The results show that we found metric invariance but were unable to find scalar invariance in the analysis of both the first and second half. Moving to the second step, we did find partial scalar 
invariance in both halves. However, the modifications for partial invariance were not reproducible. Following the process outlined in our methods, the selected intercepts freed for the partial model are not the same. That is, in examining the mis-specified parameter, freeing one parameter at a time, starting with the largest value and then checking the comparative fit for each parameter freed until scalar invariance was achieved, the first half of the data required the additional modification of freeing the third item. 


\section{Table 4}

Measurement invariance testing for Subjective well-being HALF 1

\begin{tabular}{|c|c|c|c|c|c|c|c|c|c|}
\hline Partial Invariance (SWB_2 1, SWB_3 1, SWB_4 1) & $\chi^{2}$ & df & $\chi 2$ diff & $\Delta \mathbf{d f}$ & sig & RMSEA & SRMR & CFI & TLI \\
\hline Equal form & 52.02 & 25 & & & & 0.052 & 0.016 & 0.993 & 0.987 \\
\hline Equal factor loadings & 85.72 & 41 & 33.70 & 16 & 0.006 & 0.052 & 0.038 & 0.989 & 0.986 \\
\hline \multicolumn{10}{|l|}{$\begin{array}{l}\text { Measurement invariance testing for Subjective well-being } \\
\text { HALF } 2\end{array}$} \\
\hline Partial Invariance (SWB_2 1, SWB_4 1) & $\chi^{2}$ & df & $\chi 2$ diff & $\Delta \mathbf{d f}$ & sig & RMSEA & SRMR & CFI & TLI \\
\hline Equal factor loadings & 96.09 & 37 & 54.69 & 16 & 0.000 & 0.065 & 0.046 & 0.985 & 0.980 \\
\hline Equal indicator intercepts & 299.34 & 57 & 203.25 & 20 & 0.000 & 0.136 & 0.074 & 0.938 & 0.946 \\
\hline
\end{tabular}


For the Alignment optimization, we first checked the overall degree of non-invariance for both halves. As mentioned above, the existing guidelines are that $25 \%$ non- invariance is problematic or more strictly that those above $20 \%$ require a closer look. The degree of noninvariance for the two halves was equivalent at $10 \%$ non-invariant parameters. That is, in the examination of Table 16, the number of parameters flagged as non-invariant (those in the parentheses) are 5 out of 50 for both halves. However, there are small differences regarding which parameters were flagged as non-invariant in the two halves, 3 out 5 being the same (see Table 5). The literature is not clear on the weight to give these small differences. As for the conclusion based on these results Alignment optimization is the same for both halves: There is only $10 \%$ non-invariant parameters and therefore below the acceptable threshold,.

\section{Table 5}

Alignment measurement (non) invariance for intercepts and loadings for Subjective well-being HALF

\begin{tabular}{cccccc}
\multicolumn{1}{l}{ Intercepts/Thresholds } & \multicolumn{1}{l}{} \\
\hline SWB_1 & 1 & 2 & 3 & 4 & 5 \\
SWB_2 & 1 & $(2)$ & $(3)$ & 4 & 5 \\
SWB_3 & 1 & 2 & 3 & $(4)$ & 5 \\
SWB_4 & 1 & 2 & 3 & $(4)$ & 5 \\
SWB_5 & 1 & 2 & 3 & 4 & 5 \\
\hline Loadings & & & 3 & 4 & 5 \\
\hline SWB_1 & 1 & 2 & 3 & 4 & 5 \\
SWB_2 & 1 & 2 & 3 & 4 & 5 \\
SWB_3 & 1 & 2 & 3 & 4 & 5 \\
SWB_4 & 1 & 2 & & 4 & 5 \\
SWB_5 & 1 & 2 & & &
\end{tabular}

Alignment measurement (non) invariance for intercepts and loadings for Subjective well-being HALF $\underline{2}$

\begin{tabular}{cccccc}
\hline Intercepts/Thresholds & \multicolumn{5}{c}{} \\
\hline SWB_1 & 1 & 2 & 3 & 4 & 5 \\
SWB_2 & $(1)$ & $(2)$ & 3 & 4 & 5 \\
SWB_3 & 1 & 2 & 3 & $(4)$ & 5 \\
SWB_4 & 1 & $(2)$ & 3 & $(4)$ & 5 \\
SWB_5 & 1 & 2 & 3 & 4 & 5 \\
\hline Loadings & & & 3 & 4 & 5 \\
\hline SWB_1 & 1 & 2 & 3 & 4 & 5 \\
SWB_2 & 1 & 2 & 3 & 4 & 5 \\
SWB_3 & 1 & 2 & 3 & 4 & 5 \\
SWB_4 & 1 & 2 & &
\end{tabular}


To examine how the differences in the invariance testing (such as freeing different parameters or finding different parameters invariant) may change the interpretation of the data, we also compared means based on the partial invariance and Alignment optimization (Table 6). The results show that the mean differences between countries are reproducible for both halves when applying the Alignment method. However, in the MG-CFA analysis, half 2 shows USA has significantly lower subjective well-being compared to Netherlands and India, whereas half 1 does not. Therefore, using the MG-CFA method and partial invariance, the results of the mean differences between country level subjective well-being are not reproducible. 
Table 6

Mean comparisons split half Subjective well-being

\begin{tabular}{|c|c|c|c|c|c|c|c|c|}
\hline & \multicolumn{2}{|c|}{ RAW-Composite } & \multicolumn{3}{|l|}{ MG-CFA } & \multicolumn{3}{|c|}{ ALIGNMENT } \\
\hline & & $\begin{array}{l}\text { Factor } \\
\text { Mean }\end{array}$ & $\begin{array}{l}\text { SWB_2 1, } \\
\text { SWB_3 1, } \\
\text { SWB_4 1 }\end{array}$ & $\begin{array}{l}\text { Factor } \\
\text { Mean }\end{array}$ & $\begin{array}{l}\text { Groups with } \\
\text { Significantly } \\
\text { Smaller } \\
\text { Factor Mean }\end{array}$ & & $\begin{array}{l}\text { Factor } \\
\text { Mean }\end{array}$ & $\begin{array}{l}\text { Groups with } \\
\text { Significantly } \\
\text { Smaller } \\
\text { Factor Mean }\end{array}$ \\
\hline \multirow{8}{*}{ HALF 1} & 4. & & 4. & & & & & \\
\hline & Netherlands & 5.05 & Netherlands & 0.058 & 1,2 & $\begin{array}{l}\text { 3. India } \\
4 .\end{array}$ & 0.189 & 1,2 \\
\hline & 5. USA & 5.01 & 3. India & 0.059 & 1,2 & Netherlands & 0.106 & 1,2 \\
\hline & 3. India & 4.85 & 5. USA & $\begin{array}{c}0.000 \\
-\end{array}$ & 1,2 & 5. USA & $\begin{array}{c}0.000 \\
-\end{array}$ & 1,2 \\
\hline & 1. Canada & 4.61 & 1. Canada & 0.419 & 2 & 1. Canada & 0.203 & 2 \\
\hline & 2. China & 3.61 & 2. China & 1.014 & & 2. China & 1.157 & \\
\hline & \multicolumn{2}{|c|}{ RAW-Composite } & MG-CFA & \multicolumn{5}{|c|}{ ALIGNMENT } \\
\hline & & $\begin{array}{l}\text { Factor } \\
\text { Mean }\end{array}$ & $\begin{array}{l}\text { SWB_2 } \sim 1, \\
\text { SWB_4 1 }\end{array}$ & $\begin{array}{l}\text { Factor } \\
\text { Mean }\end{array}$ & $\begin{array}{l}\text { Groups with } \\
\text { Significantly } \\
\text { Smaller } \\
\text { Factor Mean }\end{array}$ & & $\begin{array}{l}\text { Factor } \\
\text { Mean }\end{array}$ & $\begin{array}{l}\text { Groups with } \\
\text { Significantly } \\
\text { Smaller } \\
\text { Factor Mean }\end{array}$ \\
\hline \multirow{5}{*}{ HALF 2} & $\begin{array}{l}\text { 3. India } \\
4 .\end{array}$ & 5.09 & $\begin{array}{l}4 . \\
\text { Netherlands }\end{array}$ & 0.273 & $1,2,5$ & $\begin{array}{l}4 . \\
\text { Netherlands }\end{array}$ & 0.046 & 1,2 \\
\hline & Netherlands & 5.04 & 3. India & 0.271 & $1,2,5$ & 5. USA & 0.000 & 1,2 \\
\hline & 5. USA & 4.93 & 5. USA & 0.000 & 1,2 & 3. India & 0.090 & 1,2 \\
\hline & 1. Canada & 4.73 & 1. Canada & 0.168 & 2 & 1. Canada & $\begin{array}{c}0.386 \\
-\end{array}$ & 2 \\
\hline & 2. China & 3.49 & 2. China & -1.07 & & 2. China & 1.027 & \\
\hline
\end{tabular}




\section{Discussion}

In cross-cultural research, measurement invariance is used as a tool to ensure equivalence. Practitioners use the selected method to test the comparability of a scale so that any observed effect is due to the "true score" of a measured variable, not the measurement itself. Theoretically, general conclusions regarding measurement invariance should be consistent as main results are reproducible. In this study, we found this to be true when using the Alignment method, but not when using the partial invariance with MG-CFA. The process of partial invariance with MG-CFA for two randomly selected halves of the data set did not reveal reproducible results. This was the case in the process of freeing parameters and in the conclusions regarding the mean difference between countries. For the Alignment method, the conclusions regarding the mean difference were the same for the two halves. This demonstrates an advantage of Alignment optimization over the traditional MG-CFA approach. However, as with study 1, although there are advantages of doing this demonstration with real data, these conclusions need to be confirmed and supported with simulation data.

Finally, the output of the Alignment method was also not identical for the two halves. That is, there were some differences regarding which parameters were identified as noninvariant. There is very little research regarding the significance of these findings. Future research should investigate further if differences in identified non-invariant parameters lead to differing conclusions, however, in our example the conclusions were reproducible.

\section{Study 3}

The previous two studies point to the advantages of the Alignment method in simple mean comparisons. However, the primary disadvantage and obstacle for practitioners of cross-cultural research is what to do when MG-CFA finds non-invariance in a measure needed for a primary analysis. The research discusses different causes of non-invariance and 
theory dictates that if in fact measures are not comparable, we should not compare them. However, as outlined above, the traditional MG-CFA can be too strict, especially for a large number of groups. Alignment optimization suggests that approximate invariance is sufficient as long as we take into account such approximation. So far, what is missing in the literature is the application of those adjustments in investigations of research questions of interest. . In the field of cross-cultural psychology, the goal of analysis usually goes beyond a comparison of means. Measurement invariance is either found (or not) and the subsequent analysis is valid or it is not. Therefore, the application of approximate invariance is useful as it allows for some flexibility. However, it requires additional steps. In study 3, we practically demonstrate the difference between the methods by using downstream applications.

Although Alignment optimization is a good alternative to MG-CFA, there is a misconception in the literature about its implementation in research. As Marsh et al. (2018) point out, it is seen as "primarily as an exploratory tool useful in preliminary analyses". Seeing this shortfall, Marsh et al. (2018) introduced the next steps in the application of Alignment optimization. The alignment-within-CFA (AwC) approach allows for the Alignment method to be used as a confirmatory tool. While Marsh et al. (2018) applied this to a comparison of gender and culture, we will apply AwC to a dataset from the social/political psychology literature, which investigated the relationship between moral foundations and political ideologies (Graham, Haidt, \& Nosek, 2009), an effect that has also been included in the "Many Labs 2" replication project (Klein et al., 2018) and tested across countries. Within the Many Labs 2 project, this finding was suitable because it includes a Likert type measure with three or more items and a simple correlational finding that can add to the literature of examples of AwC. Importantly, the finding that people on the political left endorse individualizing foundations more than people on the right and people on the right endorsed binding foundations more than people on the left could be replicated in this project. However, Klein et al. (2018) did not check for measurement invariance in their main analysis. 
Therefore, we will compare our analysis to theirs to highlight differences in interpretation of results when the comparability of measurement is taken into account. This demonstration is intended to show how the extension of the Alignment method, AwC, can be used in practice when MG-CFA concludes non-invariance.

\section{Sample}

Utilizing the Many Labs 2 data set, this study looks at finding number 4. Moral foundations of liberals versus conservatives (Graham, Haidt, \& Nosek, 2009, Study 1). This study looks at the correlation between the moral foundation and political ideology. We included all the country groups that completed this study. The 30 countries can be seen in table $7^{3}$.

Table 7

Sample information Study 3

\begin{tabular}{llccccc}
\hline Country & $\begin{array}{l}\text { Sample } \\
\text { size }\end{array}$ & \multicolumn{3}{c}{ Binding foundation } & $\begin{array}{l}\text { Individualizing } \\
\text { foundations }\end{array}$ \\
\hline & & Mean & $\begin{array}{c}\text { Std. } \\
\text { Deviation }\end{array}$ & Mean & $\begin{array}{c}\text { Std. } \\
\text { Deviation }\end{array}$ \\
\hline 1 & Austria & 123 & 3.58 & 0.67 & 5.03 & 0.74 \\
2 & Belgium & 110 & 3.92 & 0.68 & 4.86 & 0.69 \\
3 & Brazil & 103 & 3.63 & 0.84 & 4.91 & 0.88 \\
4 & Canada & 601 & 3.98 & 0.75 & 4.82 & 0.81 \\
5 & Chile & 155 & 4.35 & 0.68 & 5.33 & 0.56 \\
6 & China & 392 & 3.95 & 0.77 & 4.14 & 0.84 \\
7 & Costa Rica & 103 & 4.22 & 0.69 & 5.21 & 0.63 \\
8 & Czach Republic & 141 & 3.96 & 0.64 & 4.75 & 0.64 \\
9 & France & 44 & 3.50 & 0.87 & 4.02 & 1.29 \\
10 & Germany & 91 & 3.58 & 0.68 & 4.99 & 0.67 \\
11 & Hong Kong & 173 & 3.91 & 0.76 & 4.55 & 0.92 \\
12 & Hungry & 182 & 3.83 & 0.70 & 5.06 & 0.67 \\
13 & India & 360 & 3.83 & 0.89 & 4.02 & 1.10 \\
14 & Japan & 114 & 3.91 & 0.69 & 4.62 & 0.68 \\
15 & Mexico & 144 & 4.12 & 0.72 & 5.21 & 0.59 \\
16 & New Zealand & 102 & 3.89 & 0.79 & 4.86 & 0.77
\end{tabular}

\footnotetext{
${ }^{3}$ This study includes samples from places like China, in which the political ideology question of describing one's self as right-wing or left-wing does not fit the cultural context, if we were to design this study ourselves we would either exclude such countries or ask the question in a different way. However, because we want our analysis to be comparable to the original we included all countries.
} 


\begin{tabular}{|c|c|c|c|c|c|c|}
\hline 17 & Poland & 231 & 4.07 & 0.63 & 4.91 & 0.48 \\
\hline 18 & Portugal & 36 & 3.97 & 0.75 & 5.03 & 0.65 \\
\hline 19 & Serbia & 107 & 3.91 & 0.78 & 5.17 & 0.54 \\
\hline 20 & South Africa & 74 & 4.01 & 0.74 & 4.96 & 0.82 \\
\hline 21 & Spain & 54 & 4.28 & 0.76 & 4.75 & 0.73 \\
\hline 22 & Sweden & 113 & 3.38 & 0.79 & 4.78 & 0.82 \\
\hline 23 & Switzerland & 109 & 3.50 & 0.81 & 4.36 & 0.94 \\
\hline 24 & $\begin{array}{l}\text { Taiwan } \\
\text { The }\end{array}$ & 137 & 4.08 & 0.80 & 4.70 & 0.71 \\
\hline 25 & Netherlands & 486 & 3.76 & 0.60 & 4.75 & 0.68 \\
\hline 26 & Turkey & 239 & 3.70 & 0.81 & 4.66 & 0.83 \\
\hline 27 & UK & 142 & 3.92 & 0.69 & 5.04 & 0.65 \\
\hline 28 & UAE & 92 & 4.13 & 0.71 & 4.82 & 0.81 \\
\hline 29 & Uruguay & 87 & 4.06 & 0.70 & 5.08 & 0.69 \\
\hline 30 & USA & 2382 & 4.08 & 0.72 & 4.93 & 0.72 \\
\hline & Total & 6896 & 3.95 & 0.75 & 4.79 & 0.82 \\
\hline
\end{tabular}

\section{Measures}

The moral foundation scale includes five subscales (each 3 items) grouped into two categories: individualizing foundations, which emphasized concerns of harm and fairness (6 items), and binding foundations, which emphasized concerns for the in-group, authority, and purity (9 items). Participants rated the relevance to moral judgment of each item on a 6-point scale from 'not at all relevant' to 'extremely relevant'.

\section{Analysis Strategy}

For the first step of testing for measurement invariance, we followed the sample analysis plan as outlined in Studies 1 and 2 for the MG-CFA and Alignment method. For Alignment optimization, we continued with the FIXED model, with Germany as the reference group.

For the next step we use the Alignment-within-CFA (AwC) approach (Marsh et al., 2018) to apply the Alignment optimization results to the research questions. AwC takes the approximations of the alignment into account and reconfigures them into a standard CFA model. Therefore, the resulting model has the same solution as the Alignment optimization in terms of number of estimated parameters, goodness of fit, and definition of the factor 
structure. We adapted the procedure of Marsh et al. (2018; Study 1) to fit our model. This step required specifying our model, taking the first output into account. The full input and output can be found in SOM.

Finally, to examine the comparability of the conclusions regarding the heterogeneity across countries, we used tests of the heterogeneity metaphor package in R (Viechtbauer, 2010,2015). Specifically, we examined the difference in the established heterogeneity estimates Q, $I^{2}$, and tau for both the individualizing foundation and binding foundation, taking into account Alignment optimization adjustments and without the adjustments.

\section{Results}

The results of the MG-CFA invariance testing can be seen in Tables 8 . The results show that we did not find metric invariance nor scalar invariance ${ }^{4}$.

For the Alignment optimization, we first checked the overall degree of non-invariance. The degree of non-invariance was $10 \%$ non-invariant parameters (see Table S12 in SOM), which is well within the guidelines (Information about the comparison of means can be found in $\mathrm{SOM})$.

Next, we looked at the conclusions drawn with regard to the question at hand when applying AwC. We compared the correlations between the key variables when the Alignment optimization adjustment were applied to those from the raw data, and considered what conclusions would be drawn with regard to the significance of the correlation, when testing the significance value at $p<0.05$. The overall correlation between moral foundations and political ideology with and without alignment corrections can be seen in Table 9. In this

\footnotetext{
${ }^{4}$ An examination of the invariant parameters using the same procedure described in studies 1 and 2 showed that it was necessary to allow at least one item (i.e., Graham_3 in our analysis) to load freely across groups in order to lower the delta CFI to .009 , achieving metric invariance. As the original analysis performed by Klein et al. (2018) required metric invariance on the first place, this can be regarded already as problematic in the reproduction of their results. However, we performed all further analyses using raw means without excluding any items in order to compare our results directly to those from the original study.
} 
analysis the conclusions are the same: individualizing foundations are significantly negativity correlated with political ideology and binding foundation are significantly positively correlated with political ideology. However, in the analysis at the individual country level we find some variability in the robustness of findings. While for 25 countries the conclusions remained the same, for five countries (Germany, Mexico, Sweden, UK, and Uruguay) they do not, (in the case of Sweden a difference of significance level). For example, in the Germany sample the negative correlation between individualizing foundations and political ideology does not reach significance when the analysis does not take the alignment adjustments into account (see Table 10). Therefore, if these adjustments are not applied one might conclude that there is not a significant relationship between individualizing foundations and political ideology in Germany. For all country level correlations see SOM (S13a-dd). 
Table 8

Measurement invariance testing for Moral Foundation.

\begin{tabular}{|c|c|c|c|c|c|c|c|c|c|}
\hline & $\chi^{2}$ & df & $\chi 2$ diff & $\Delta \mathbf{d f}$ & sig & $\begin{array}{c}\text { RMSEA } \\
\text { (90\% } \\
\text { CI }) \\
\end{array}$ & SRMR & CFI & TLI \\
\hline Equal form & 6626.72 & 2670 & & & & 0.082 & 0.065 & 0.850 & 0.823 \\
\hline Equal factor loadings & 7281.75 & 3047 & 655.03 & 377.00 & 0.00 & 0.079 & 0.077 & 0.840 & 0.834 \\
\hline Equal indicator intercepts & 11040 & 3424 & 3758.30 & 377.00 & 0.00 & 0.100 & 0.097 & 0.711 & 0.735 \\
\hline
\end{tabular}




\section{Table 9}

Study 3 correlations between moral foundations and political ideology

\begin{tabular}{|c|c|c|c|}
\hline & $\begin{array}{l}\text { Individualizing } \\
\text { foundations }\end{array}$ & $\begin{array}{l}\text { Binding } \\
\text { foundation }\end{array}$ & $\begin{array}{l}\text { Political } \\
\text { Ideology }\end{array}$ \\
\hline $\begin{array}{l}\text { Individualizing } \\
\text { foundations }\end{array}$ & 1 & & \\
\hline $\begin{array}{l}\text { Binding } \\
\text { foundation }\end{array}$ &, $483 * * / 699 * *$ & 1 & \\
\hline $\begin{array}{l}\text { Political } \\
\text { Ideology }\end{array}$ &,$- 134 * * /-.104 * *$ &, $145 * * / .145 * *$ & 1 \\
\hline
\end{tabular}

\section{Table 10}

Study 3 correlations between moral foundations and political ideology in Germany

\begin{tabular}{|c|c|c|c|}
\hline & $\begin{array}{l}\text { Individualizing } \\
\text { foundations }\end{array}$ & $\begin{array}{l}\text { Binding } \\
\text { foundation }\end{array}$ & $\begin{array}{l}\text { Political } \\
\text { Ideology }\end{array}$ \\
\hline $\begin{array}{l}\text { Individualizing } \\
\text { foundations }\end{array}$ & 1 & & \\
\hline Binding foundation & $.806 * * / .732 * *$ & 1 & \\
\hline Political Ideology & $-.188 /-.216^{*}$ & $.115 / .159$ & 1 \\
\hline
\end{tabular}

The second research question of Main Labs 2 regarded the heterogeneity across sample locations. This analysis determines the amount of variation that exceeds expected measurement error. As the design is equal across locations the detected variation can be attributed to sample differences. According to our results on the variability in the binding foundations, as seen in estimates of heterogeneity, they are the same regardless of whether alignment adjustments are taken into account. For this scale the tau, $Q$ test and $I^{2}$ are almost indistinguishable in the two analyses. In both analyses the tau values are equivalent, while the $Q$ test shows significant heterogeneity and the $I^{2}$ statistic indicates medium heterogeneity. The individualizing foundations however showed some variability between analyses. While the 
tau value is almost equivalent and the $I^{2}$ values both indicate moderate heterogeneity, the $Q$ values would lead to different conclusions. With the alignment adjustments the value of the $Q$ test reaches significance and without these adjustments, it does not. Therefore, the analysis of variation across sample countries shows that, if measurement invariance in not considered, one would conclude that there is insignificant heterogeneity, however, when measurement invariance is required, one would conclude that there is significant heterogeneity. Table 11 summarizes these results.

\section{Table 11}

Study 3 Results of Heterogeneity Tests

$$
\text { tau }
$$

\begin{tabular}{|c|c|c|c|c|c|}
\hline & $\mathrm{au}$ & Q & $d f$ & $p$ & $I^{2}$ \\
\hline \multicolumn{6}{|l|}{ Individualizing foundations } \\
\hline $\begin{array}{l}\text { Without Alignment optimization } \\
\text { adjustments }\end{array}$ & 0.05 & 41.05 & 29 & 0.068 & $34.48 \%$ \\
\hline $\begin{array}{l}\text { With Alignment optimization } \\
\text { adjustments }\end{array}$ & 0.06 & 46.06 & 29 & 0.023 & $39.50 \%$ \\
\hline \multicolumn{6}{|l|}{ Binding foundation } \\
\hline $\begin{array}{l}\text { Without Alignment optimization } \\
\text { adjustments } \\
\text { With Alignment optimization }\end{array}$ & 0.08 & 54.81 & 29 & 0.003 & $52.28 \%$ \\
\hline adjustments & 0.08 & 54.52 & 29 & 0.003 & $51.84 \%$ \\
\hline
\end{tabular}

\section{Discussion}

This study built upon the previous two studies as we did not only compare the two methods in question, but we demonstrated the utility of the Alignment optimization method extension $\mathrm{AwC}$ and highlighted the differences in the interpretation of key results when this method is applied. . As Marsh et al. (2018) explained: "if there is good support for complete scalar invariance, there is no need to pursue alignment. However, in large-scale studies, scalar CFA-MI models are almost always rejected.” (p. 2). This key observation was demonstrated and built upon in this study. Across 30 countries scalar invariance could not be established and therefore, the Alignment optimization method was necessary to complete the analysis.

The goal of original research, as with many cross-cultural research projects, is to move beyond a comparison of means. In this study, after finding that using Alignment optimization 
was more suitable, we took the next analytical steps. With regard to our main research question focusing on the correlation between moral foundations and political ideology, we came to the same conclusions as the original study, both with and without alignment adjustments. However, this was not always the case when looking at the results by sample country. In this analysis, five countries differed in the conclusion when the Alignment adjustments were applied. As for the secondary question, the replication of the heterogeneity of finding across samples, we found generally reproducible results. However, one estimate, the $Q$ test did vary between our results that take into account measurement invariance and those that do not. This means, if one uses the $Q$-value to determine whether there is heterogeneity across samples, a different conclusion would be drawn in the two analyses: It indicated significant variation between samples only when the alignment modifications were considered. Therefore, results are not fully reproducible.

Although the overall conclusions of the analysis were comparable, this study highlights two key points. First, it is vital to remember that without alignment adjustments, results from different groups (in this case, countries) should not be compared. From a basic theorical standpoint, this means comparing apples and oranges. In the case analyzed here, the differences were minimal, however, it is still necessary to take such comparisons into account. Second, we choose a rather stable finding in a well-designed replication project therefore reproducibility was expected. That is, in the Many Labs 2, the effect size was reportedly large in the original study (.52) and medium in their replication (.23) and the scales have previously been used in cross-cultural research, including testing for invariance (Doğruyol, Alper, \& Yilmaz, 2019, Iurino, \& Saucier, 2020). Therefore, this was a generally robust test of the application of the method. Other research materials and designs might be more problematic than the ones included in the current research, making the use of alignment method and AwC even more necessary for a valid comparison.

\section{General Discussion}


Although the "Apples to Oranges" problem of comparing non-equivalent measurements is widely known in cross-cultural research and beyond, there is still a lack of action to rectify the lack of testing. One road block to the lack of reported invariance testing is the question of what to do when traditional methods find non-invariance. This paper outlined the comparison of traditional MG-CFA and the newer Alignment optimization method. Beyond describing the different methods, we included three demonstrative examples of how these methods can be applied in practice.

First, in Study 1, which used a moderate number of groups (eight countries), we showed the limitations of MG-CFA and partial invariance because it requires some countries to be excluded from analysis while the Alignment method produced a valid analysis without excluding countries. Further, we showed the difference in methods mattered in the downstream conclusions taken from the data in the comparison of the mean values. Nonetheless, it is important to note that simulation studies are required for a definitive comparison of the accuracy of the two methods; Marsh et al. (2018) is a good example of this. However, it is still valuable to see the differences cross-cultural researchers can encounter in the research process.

Next, Study 2 took a similar approach by using real data (again with a moderate number of groups) to test the reproducibility of findings. We found that the Alignment optimization results regarding the conclusions of the invariance test and the mean differences between countries were the same for half 1 and half 2. However, with MG-CFA the partial invariance procedure did lead to the freeing of different parameters, as predicted by the critics of the approach (Marsh et al 2018). Furthermore, the analysis of the two halves led to different conclusions with regards to mean differences between groups. Therefore, the results were reproducible in a cross-validation analysis with Alignment optimization method but not for MG-CFA. 
Finally, Study 3 provides practical answers for questions form practitioners of crosscultural research: What does one do when scalar invariance fails but the analysis requires it? Given that we showed the drawbacks of the restrictiveness of MG-CFA, the good news is that Alignment optimization offers a viable alternative. Furthermore, this study demonstrated the utility of AwC. Whereas MG-CFA is a gate that either finds invariance or not, Alignment optimization is an approximation that allows for further analysis needs to take this approximation into account as is done with $\mathrm{AwC}$. This is paper is a rare example of how this can be done.

Importantly, we show that $\mathrm{AwC}$ delivers results which differ partially from those found without it regarding 1) the effect in some of the countries and 2) the overall heterogeneity of the effects in some of the indicators. Thus, one important implication is that it is not always the case that results are non-replicable (as someone could say about the findings in the German sample), but we might be observing some false negatives due to nonoptimal methods ${ }^{5}$. Thus, in the age of the replication crisis, this study shows the practical value of AwC to the study of cross-cultural phenomena.

\section{Limitations}

A limitation to the method in this study is the sample size and sample makeup. Firstly, in methods validation, larger sample sizes are sometimes recommended for MI testing (Meade, 2005; Meade \& Lautenschlager, 2004). Secondly, to be able to make a definitive statement about the country level means, it is necessary to have a representative sample, whereas our sample consists of mostly young adults and students. However, the samples we obtained are representative for cross-cultural research (Shen et al. 2011). Often, when involving many different research groups in a project, there is a disparity in what collaborators deliver, and less than ideal sample sizes is the norm. Although a majority of

\footnotetext{
${ }^{5}$ Our reproduction attempt using AwC also unveiled evidence for type I error in some samples (i.e., we could not reproduce the results). Effects in all countries can be found in the SOM.
} 
undergraduates in the samples is a limitation in our conclusions about country level means, having sample groups that are similar in age and education and only differ in nationality leads to more conclusive findings with regard to the equivalence of the measurement scales used. Furthermore, both of these points are strengths as well as weaknesses because they represent common research practice, and lie at the heart of our question, which is the utility of methods for use in common research. Future research should both replicate these findings and compare them to further alternative methods.

An additional caveat to all invariance testing is that it can only statistically ensure statistical equivalence. Even if we can establish strict MI, confirming that there is no bias in factor loadings and item intercepts across countries, we cannot guarantee conceptual equivalence or, in other words, lack of construct bias. The whole construct could differ in meaning across cultures. MI does not necessarily mean lack of all types of bias. For example, bias could influence all the indicators to the same degree, therefore having a biased estimation of the latent mean. Future research should investigate how to theoretically identify measurement bias that is not statistically identifiable.

\section{Conclusion}

Establishing measurement invariance is necessary for the comparison of homogeneous groups. However, it is often overlooked. Research has highlighted the limitations of the MGCFA for large scale studies and opened the door to exploring new methods for testing MI. Past solutions, such as limiting the countries in analysis of partial invariance, are insufficient. This article demonstrates the strengths of the Alignment optimization method compared to MG-CFA: it is more flexible and therefore more likely to find invariance without the multiple corrections required with partial invariance, and likely still has less biased latent means. Furthermore, we found the MG-CFA and partial invariance showed consistently less reproducible results than Alignment optimization and $\mathrm{AwC}$. This difference in itself supports 
the argument that MG-CFA has many significant limitations. Future research should replicate these findings and further explore what this means on a conceptual level. 


\section{References}

Altemeyer, B. (1981). Right-wing authoritarianism. Winnipeg, Canada: University of Manitoba Press

Altemeyer, B., \& Hunsberger, B. (1992). Authoritarianism, religious fundamentalism, quest, and prejudice. The international journal for the psychology of religion, 2, 113-133.

Asparouhov, T., \& Muthén, B. O. (2014). Multiple-group factor analysis alignment. Structural Equation Modeling: A Multidisciplinary Journal, 1-14.

Boer, D., Hanke, K., \& He, J. (2018). On detecting systematic measurement error in crosscultural research: A review and critical reflection on equivalence and invariance tests. Journal of Cross-Cultural Psychology, 49, 713-734.

Byrne, B. M., \& van de Vijver, F. J. (2017). The maximum likelihood alignment approach to testing for approximate measurement invariance: A paradigmatic cross-cultural application. Psicothema, 29.

Byrne, B. M., Shavelson, R. J., \& Muthén, B. (1989). Testing for the equivalence of factor covariance and mean structures: The issue of partial measurement invariance. Psychological Bulletin, 105, 456-466.

Chen, F. F. (2007). Sensitivity of goodness of fit indexes to lack of measurement invariance. Structural Equation Modeling: A Multidisciplinary Journal, 14, 464-504.

Chen, F. F. (2008). What happens if we compare chopsticks with forks? The impact of making inappropriate comparisons in cross-cultural research. Journal of Personality and Social Psychology, 95, 1005-1018.

Cheung, G. W., \& Rensvold, R. B. (2000). Assessing extreme and acquiescent response sets in cross-cultural research using structural equations modeling. Journal of CrossCultural Psychology, 31, 187-212.

Cheung, G. W., \& Rensvold, R. B. (2002). Evaluating goodness-of-fit indexes for testing measurement invariance. Structural Equation Modeling, 9, 233-255. 
Cieciuch, J., Davidov, E., Algesheimer, R., \& Schmidt, P. (2018). Testing for approximate measurement invariance of human values in the European Social Survey. Sociological Methods \& Research, 47, 665-686.

Cohen, J. (1994). The earth is round (p<. 05). In What if there were no significance tests? (pp. 69-82). Routledge.

Davidov, E., Dülmer, H., Schlüter, E., Schmidt, P., \& Meuleman, B. (2012). Using a multilevel structural equation modeling approach to explain cross-cultural measurement noninvariance. Journal of Cross-Cultural Psychology, 43, 558-575.

Davidov, E., Meuleman, B., Cieciuch, J., Schmidt, P., \& Billiet, J. (2014). Measurement equivalence in cross-national research. Annual Review of Sociology, 40, 55-75.

Davidov, E., Schmidt, P., \& Schwartz, S. H. (2008). Bringing values back in: The adequacy of the European Social Survey to measure values in 20 countries. Public opinion quarterly, 72, 420-445.

Doğruyol, B., Alper, S., \& Yilmaz, O. (2019). The five-factor model of the moral foundations theory is stable across WEIRD and non-WEIRD cultures. Personality and Individual Differences, 151.

Duckitt, J., \& Sibley, C. G. (2007). Right wing authoritarianism, social dominance orientation and the dimensions of generalized prejudice. European Journal of Personality: Published for the European Association of Personality Psychology, 21, 113-130.

Graham, J. W. (2009). Missing data analysis: Making it work in the real world. Annual review of psychology, 60, 549-576.

Hofstede, G. (1980a). Culture's Consequences: International Differences in Work-Related Values. Beverly Hills, CA: Sage.

Hofstede, G., Hofstede, G. J. \& Minkov, M. (2010). Cultures and Organizations: Software of the Mind (Rev. 3rd ed.). New York: McGraw-Hill. 
Heath, A., Martin, J., \& Spreckelsen, T. (2009). Cross-national Comparability of Survey Attitude Measures. International Journal of Public Opinion Research, 21, 293-315.

Ho, A. K., Sidanius, J., Kteily, N., Sheehy-Skeffington, J., Pratto, F., Henkel, K. E., Foels, R., \& Stewart, A. L. (2015). The Nature of Social Dominance Orientation: Theorizing and Measuring Preferences for Intergroup Inequality Using the New SDO7 Scale. Journal of Personality and Social Psychology, 109, 1003-1028.

Inglehart, R., C. Haerpfer, A. Moreno, C. Welzel, K. Kizilova, J. Diez-Medrano, M. Lagos, P. Norris, E. Ponarin \& B. Puranen et al. (eds.). 2014. World Values Survey: Round Six Country-Pooled Datafile Version: http://www.worldvaluessurvey.org/WVSDocumentationWV6.jsp. Madrid: JD Systems Institute.

Inglehart, R., C. Haerpfer, A. Moreno, C. Welzel, K. Kizilova, J. Diez-Medrano, M. Lagos, P. Norris, E. Ponarin \& B. Puranen et al. (eds.). 2014. World Values Survey: Round Five Country-Pooled Datafile Version: www.worldvaluessurvey.org/WVSDocumentationWV5.jsp. Madrid: JD Systems Institute.

Iurino, K., \& Saucier, G. (2020). Testing measurement invariance of the Moral Foundations Questionnaire across 27 countries. Assessment, 27(2), 365-372.

Jöreskog, K. G. (1971). Simultaneous factor analysis in several populations. Psychometrika, $36,409-426$.

Jorgensen, T. D., Pornprasertmanit, S., Schoemann, A. M., \& Rosseel, Y. (2018). semTools: Useful tools for structural equation modeling. R package version 0.5-1. Retrieved from https://CRAN.R-project.org/package=semTools

Kim, E. S., Cao, C., Wang, Y., \& Nguyen, D. T. (2017). Measurement invariance testing with many groups: A comparison of five approaches. Structural Equation Modeling: A Multidisciplinary Journal, 24, 524-544. 
Lomazzi, V. (2018). Using Alignment Optimization to Test the Measurement Invariance of Gender Role Attitudes in 59 Countries. Methods, data, analyses: a journal for quantitative methods and survey methodology (mda), 12, 77-103.

MacCallum, R. C., Roznowski, M., \& Necowitz, L. B. (1992). Model modifications in covariance structure analysis: The problem of capitalization on chance. Psychological Bulletin, 111, 490-504.

Magraw-Mickelson, Z., \& Gollwitzer, M. (2018). Relational and Group Collective Self Responses to Observed Victimization Across Cultures. Social Justice Research, 31, 113-132.

Manganelli Rattazzi, A. M., Bobbio, A., \& Canova, L. (2007). A short version of the RightWing Authoritarianism (RWA) Scale. Personality and Individual Differences, 43, 12231234.

Markus, H. R., \& Kitayama, S. (1991). Culture and the self: Implications for cognition, emotion, and motivation. Psychological review, 98, 224.

Marsh, H. W., Guo, J., Parker, P. D., Nagengast, B., Asparouhov, T., Muthén, B., \& Dicke, T. (2018). What to do when scalar invariance fails: The extended alignment method for multi-group factor analysis comparison of latent means across many groups. Psychological Methods, 23, 524-545.

Marsh, H. W., Hau, K. T., \& Wen, Z. (2004). In search of golden rules: Comment on hypothesis-testing approaches to setting cutoff values for fit indexes and dangers in overgeneralizing Hu and Bentler's (1999) findings. Structural equation modeling, 11, $320-341$.

McSweeney, B. (2002). Hofstede's model of national cultural differences and their consequences: A triumph of faith-a failure of analysis. Human relations, 55, 89-118. 
Meade, A. W. (2005, April). Sample size and tests of measurement invariance. In Annual Conference of the Society for Industrial and Organizational Psychology, Los Angeles, CA.

Meade, A. W., \& Lautenschlager, G. J. (2004). A Monte-Carlo study of confirmatory factor analytic tests of measurement equivalence/invariance. Structural Equation Modeling, $11,60-72$.

Meredith, W. (1993). Measurement invariance, factor analysis and factorial invariance. Psychometrika, 58, 525-543.

Muthén, B., \& Asparouhov, T. (2013a). BSEM Measurement Invariance Analysis. Mplus Web Notes: No. 17, January 11. (Vol. 17, p. 313). Retrieved February 2, 2017, from https://www.statmodel.com/examples/webnotes/webnote17.pdf

Muthén, B., \& Asparouhov, T. (2013b). New Methods for the Study of Measurement Invariance with Many Groups. Technical report. Available online at: http:// statmodel2.com/download/PolAn.pdf.

Muthén, B., \& Asparouhov, T. (2014). IRT studies of many groups: the alignment method. Frontiers in Psychology, 5, 978.

Muthén, L. K., \& Muthén, B. O. (1998-2018). Mplus user's guide (version 8.1). Los Angeles, CA: Muthén \& Muthén.

Perry, R., Sibley, C. G., \& Duckitt, J. (2013). Dangerous and competitive worldviews: A meta-analysis of their associations with social dominance orientation and right-wing authoritarianism. Journal of Research in Personality, 47, 116-127.

Pratto, F., Liu, J. H., Levin, S., Sidanius, J., Shih, M., Bachrach, H., \& Hegarty, P. (2000). Social dominance orientation and the legitimization of inequality across cultures. Journal of Cross-Cultural Psychology, 31, 369-409. 
Pratto, F., Sidanius, J., Stallworth, L., \& Malle, B. (1994). Social dominance orientation: A personality variable predicting social and political attitudes. Journal of Personality and Social Psychology, 67, 741-763.

Reise, S. P., Widaman, K. F., \& Pugh, R. H. (1993). Confirmatory factor analysis and item response theory: Two approaches for exploring measurement invariance. Psychological Bulletin, 114, 552-566.

Rensvold, R. B., \& Cheung, G.W. (2001). Testing for metric invariance using structural equation models: Solving the standardization problem. In C. A. Schriesheim \& L. L. Neider (Eds.), Research in management: Vol. 1. Equivalence in measurement (pp. 2150). Greenwich, CT: Information Age.

Rosseel, Y (2012). lavaan: An R Package for Structural Equation Modeling. Journal of Statistical Software, 48, 1-36. URL http://www.jstatsoft.org/v48/i02/

Rutkowski, L., \& Svetina, D. (2014). Assessing the hypothesis of measurement invariance in the context of large-scale international surveys. Educational and Psychological Measurement, 74, 31-57.

Schmitt, M., Gollwitzer, M., Maes, J., \& Arbach, D. (2005). Justice sensitivity. European Journal of Psychological Assessment, 21, 202-211.

Schmitt, M., Gollwitzer, M., Maes, J., \& Arbach, D. (2005). Justice sensitivity: Assessment and location in the personality space. European Journal of Psychological Assessment, $21,202-211$.

Shen, W., Kiger, T. B., Davies, S. E., Rasch, R. L., Simon, K. M., \& Ones, D. S. (2011). Samples in applied psychology: Over a decade of research in review. Journal of Applied Psychology, 96, 1055.

Sibley, C. G., \& Duckitt, J. (2008). Personality and prejudice: A meta-analysis and theoretical review. Personality and Social Psychology Review, 12, 248-279. 
Sörbom (1974). A general method for studying differences in factor means and factor structures between groups. British Journal of Mathematical and Statistical Psychology, 27, 229-239.

Steenkamp, J.-B. E. M., \& Baumgartner, H. (1998). Assessing measurement invariance in cross-national consumer research. Journal of Consumer Research, 25, 78-90.

Steinmetz, H. (2013). Analyzing observed composite differences across groups. Methodology. $9,1-12$.

Schwartz, S. H. (1994). Beyond individualism-collectivism: New cultural dimensions of values. In U. Kim, H. C. Triandis, C. Kagitcibasi, S. C. Choi, \& G. Yoon (Eds.), Individualism and collectivism: Theory, method and applications (pp. 85-119). Thousand Oaks, CA: Sage.

Schwartz, S. H. (2006). A theory of cultural value orientations: Explication and applications. Comparative Sociology, 5, 136-182.

Triandis, H. C. (2001). Individualism-collectivism and personality. Journal of personality, 69, 907-924.

Triandis, H. C., \& Gelfand, M. J. (1998). Converging measurement of horizontal and vertical individualism and collectivism. Journal of Personality and Social Psychology, 74, 118.

van de Schoot, R., Kluytmans, A., Tummers, L., Lugtig, P., Hox, J., \& Muthen, B. (2013). Facing off with Scylla and Charybdis: a comparison of scalar, partial, and the novel possibility of approximate measurement invariance. Frontiers in Psychology, 4, 770. van de Vijver, F., \& Tanzer, N. K. (2004). Bias and equivalence in cross-cultural assessment: an overview. Revue Européenne de Psychologie Appliquée/European Review of Applied Psychology, 54, 119-135.

Vandenberg, R. J., \& Lance, C. E. (2000). A review and synthesis of the measurement invariance literature: suggestions, practices, and recommendations for organizational research. Organizational Research Methods, 3, 4-70. 
Vandenberg, R. J. (2002). Toward a further understanding of and improvement in measurement invariance methods and procedures. Organizational research methods, 5, 139-158.

World Bank (2008). World Bank, International Comparison Program database. Retrieved 1 December 2018 from https://data.worldbank.org/indicator/NY.GNP.PCAP.PP.CD

Wu, M. S., Schmitt, M., Zhou, C., Nartova-Bochaver, S., Astanina, N., Khachatryan, N., \& Han, B. (2014). Examining self-advantage in the suffering of others: Cross-cultural differences in beneficiary and observer justice sensitivity among Chinese, Germans, and Russians. Social Justice Research, 27, 231-242.

Jang, S., Kim, E. S., Cao, C., Allen, T. D., Cooper, C. L., Lapierre, L. M., ... \& Abarca, N. (2017). Measurement invariance of the satisfaction with life scale across 26 countries. Journal of Cross-Cultural Psychology, 48, 560-576.

Flake, J. K., \& McCoach, D. B. (2018). An Investigation of the Alignment Method With Polytomous Indicators Under Conditions of Partial Measurement Invariance. Structural Equation Modeling: A Multidisciplinary Journal, 25, 56-70.

Fischer, R., Hanke, K., \& Sibley, C. G. (2012). Cultural and institutional determinants of social dominance orientation: A cross-cultural meta-analysis of 27 societies. Political Psychology, 33, 437-467.

Schimmack, U., Oishi, S., \& Diener, E. (2005). Individualism: A valid and important dimension of cultural differences between nations. Personality and Social Psychology Review, 9, 17-31. 